\title{
Mumps outbreaks across England and Wales in 2004: observational study
}

Emma Savage, Mary Ramsay, Joanne White, Stuart Beard, Heather Lawson, Rashpal Hunjan, David Brown

\section{Introduction}

In October 1988, routine immunisation against mumps was introduced in the United Kingdom as a combined measles, mumps, and rubella (MMR) vaccine for children aged 12-15 months. Although the incidence of mumps declined quickly, mathematical modelling supported the introduction of a second dose of MMR in $1996 .{ }^{1}$ In 1999, numbers of cases of mumps in teenagers in England and Wales began to rise. We report a further increase in the incidence of mumps in 2004.

\section{Participants, methods, and results}

Notifications of clinically diagnosed and laboratory confirmed mumps cases are collated for England and Wales by the Health Protection Agency Centre for Infections. Confirmed cases include those obtained by routine follow-up of all notified cases where IgM is detected in oral fluid.

In 2004, the number of mumps notifications in England and Wales increased to 16436 from 4204 in 2003. A total of 8104 cases of mumps were confirmed in 2004, compared with a total of 3907 cases in the previous five years. All regions reported more cases in 2004 than in 2003. Cases were predominantly in older teenagers and young adults born before 1987 (figure), and most were in those born between 1983 and 1986 . Many of this group are currently at tertiary level education institutions and are associated with outbreaks in universities and colleges across England and Wales. Outbreaks in the same birth cohorts have also been reported in military establishments and prisons. ${ }^{2}$ Only 2.4\% (197/8104) of confirmed cases in 2004 occurred in children who would have been offered two doses routinely-that is, those born between 1993 and 1999 (figure); only 29 of these had documented receipt of two doses of MMR. Only 62 cases $(0.8 \%)$ have been confirmed in children born after 1999.

\section{Comment}

During 2004, numbers of notifications and confirmed cases of mumps increased dramatically in all regions across England and Wales, after several years of moderately increased incidence. Most cases are in young adults born before 1988, who would not have been routinely scheduled for MMR during childhood. The highest attack rate was in those born between 1983 and 1986, who were too old to be offered MMR vaccination routinely when it was introduced in 1988 , although some may have received one dose of MMR as part of a catch-up programme offered at school entry. ${ }^{3}$ These individuals would have missed the opportunity for mumps exposure during childhood because high coverage in younger children had reduced circulation of mumps in the UK. Older individuals are more likely

\section{What is already known about this topic}

The incidence of mumps fell after the MMR was introduced in 1988

The number of confirmed mumps cases in 2004

has risen across England and Wales, with a number of reported outbreaks in universities

\section{What this study adds}

The current mumps outbreak has been predominantly in older teenagers and young adults, who would not have been offered two doses of MMR

This confirms the effectiveness of the current vaccination policy

to have had mumps when it was still a common childhood infection. The absence of cases in children in younger cohorts, including those affected by the fall in MMR coverage since $1995,{ }^{4}$ confirms the effectiveness of the current vaccination policy.

The mumps component of the MMR vaccine used in the UK is believed to offer around $90 \%$ protection for one dose, although recent reports indicate that this could be as low as $64 \% .{ }^{5}$ This outbreak confirms that the current, two dose MMR schedule is effective in preventing mumps. In addition to improving routine coverage of MMR, reviewing the vaccination status of all school leavers is recommended, to ensure that they have received two doses of MMR. Other opportunities to offer MMR vaccine to the age group at highest risk, such as when entering university or other institutions, should also be considered.

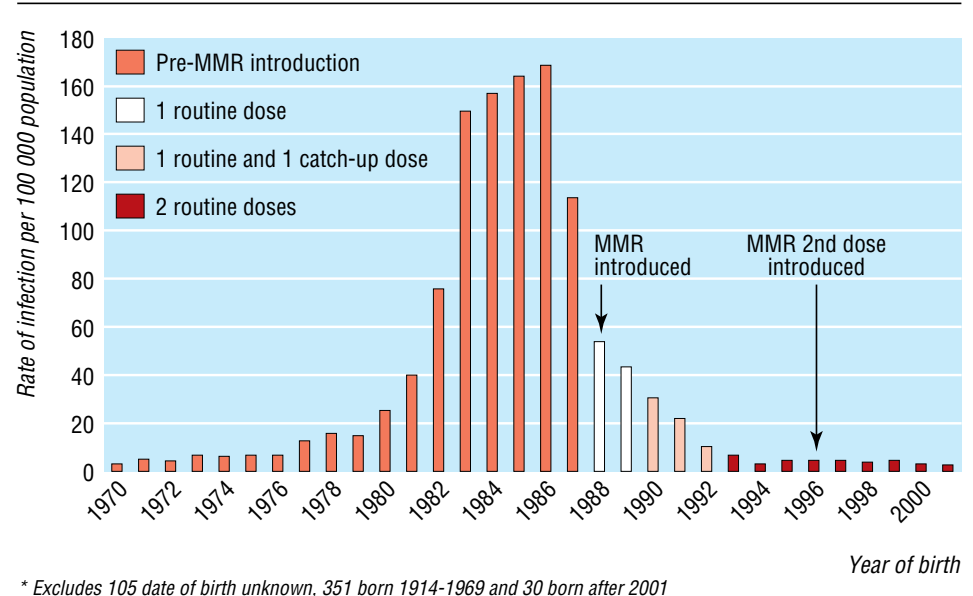

Confirmed cases of mumps in 2004 by year of birth (1970-2001) per 100000 population and opportunity for MMR vaccination (excludes 105 cases of unknown birth year, 351 born in 1914-69, and 30 born after 2001)
See also p 1120 , and Clinical review p 1132

Immunisation Department, Communicable Disease

Surveillance Centre, Health Protection

Agency, Centre for NW9 5EQ

Emma Savage scientist

Mary Ramsay consultant epidemiologist Joanne White clinical scientist

Enteric Respiratory and Neurological Virus Laboratory, Health Protection

Infections

Stuart Beard biomedical scientist Heather Lawson biomedical scientist Rashpal Hunjan biomedical scientist

David Brown consultant medical virologist

Correspondence to: E Savage Emma.Savage@ HPA.org.uk

BMJ 2005;330:1119-20 Infections, London Agency, Centre for 
Contributors: ES, MR, and JW conceived and designed the study. ES conducted the epidemiological analyses. SB, HL, RH, and DB interpreted laboratory data. All authors critically reviewed and contributed to the final draft of the paper. MR is guarantor.

Funding: None.

Competing interests: DB's laboratory has received funding from vaccine manufacturers for specific projects. He has acted as an unpaid expert witness in MMR litigation for vaccine companies. All other authors have nothing to declare.

1 Gay NJ, Miller E, Hesketh L, Morgan-Capner P, Ramsay M, Cohen B, et al. Mumps surveillance in England and Wales supports introduction of two dose vaccination schedule. Commun Dis Rep CDR Rev 1997;7(2): R21-6.

Communicable Disease Surveillance Centre. Laboratory confirmed cases of measles, mumps and rubella, England and Wales: July to Septembe 2002. Commun Dis Rep CDR Wkly 2002;12(48).

3 Gay NJ, Valambia S, Galasko D, Miller E. Selective rubella vaccination programmes: a survey of districts in England and Wales. Commun Dis Rep CDR Rev 1994;4(7):R77-9.

4 Communicable Disease Surveillance Centre. Fall in MMR vaccine coverage reported as further evidence of vaccine safety is published. Commun Dis Rep CDR Wkly 1999;9(26):227, 230.

5 Harling R, White JM, Ramsay ME, MacSween K, van den Bosch C. The effectiveness of the mumps component of the MMR vaccine: a case control study. Vaccine 2005 (in press)

(Accepted 14 April 2005)

\section{MMR vaccine and Crohn's disease: ecological study of hospital admissions in England, 1991 to 2002}

Valerie Seagroatt

See also p 1119, and Clinical review p 1132

Unit of Health-Care Epidemiology, Department of Public Health,

University of

Oxford, Oxford

OX3 7LF

Valerie Seagroatt

statistician

valerie.seagroatt@ dphpc.ox.ac.uk
It has been hypothesised that the measles, mumps, and rubella vaccine (MMR vaccine) increases the risk of autism and Crohn's disease. Although a possible link with autism has been extensively studied and refuted, ${ }^{1}$ a link with Crohn's disease has not. I tested this hypothesis by analysing trends in age specific admission rates for Crohn's disease in children and adolescents to determine if the introduction of MMR vaccine in 1988 increased rates in those populations that were offered the vaccine as infants.

\section{Methods and results}

Counts of admissions, taken as the first consultant episode in a hospital stay, in patients aged $\leq 18$ years with a main diagnosis of Crohn's disease in England (population 50 million) were available for the 12 years from April 1991 to March 2003. ${ }^{\mathrm{w} 1}$ I restricted the analysis to emergency admissions as these were probably less susceptible to changes in thresholds for admission and clinical practice than elective admissions. In the first two years of the MMR vaccination programme, the percentages of children completing a primary course of MMR vaccine in their second year of life were $7 \%$ and $68 \%$; thereafter the percentage was at least $84 \%{ }^{\text {w2 }}$

Initially, temporal trends in age specific rates were plotted, differentiating between the rates for those born before and after the introduction of the vaccine. (Rates for patients aged 16 to 18 years-too old to have been offered the vaccine as infants-provided information on underlying trends unaffected by MMR vaccination in infancy.) Data for those born in 1987-8, of whom only $68 \%$ were vaccinated as infants, were excluded from the analysis. The MMR vaccination programme was then modelled as a variable with two levels (vaccination rate of $\geq 84 \%$ and of $\leq 7 \%$ ) using Poisson regression, with adjustment for year of admission and age in single years (as categorical variables).

There were 4463 admissions for Crohn's disease, 923 of which occurred in populations with a vaccination rate of $\geq 84 \%$ (those born in 1988-9 or later). Although the age specific rates increased over the study period, no obvious changes occurred that coincided with the intro-

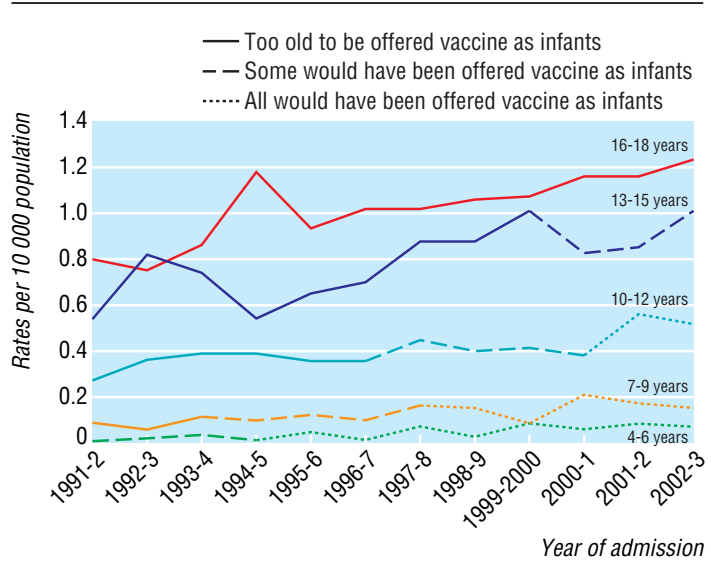

Age specific rates per 10000 population per year for emergency hospital admissions for Crohn's disease in England, 1991 to 2002. Rates in children aged $<4$ years were relatively low and so were excluded from the figure. Three-year groups, rather than the more conventional five-year groups, were used in order to discriminate between rates in children born before and after the introduction of MMR

duction of MMR vaccine (figure). The estimated rate ratio for the MMR vaccination programme (rates in populations with a vaccination rate of $\geq 84 \%$ compared with those with a rate of $\leq 7 \%$ ) was $0.95(95 \%$ confidence interval 0.84 to 1.08 )

\section{Comment}

The introduction of MMR vaccine, replacing the single measles vaccine, was not associated with an increase in Crohn's disease. Given the precision of the rate ratio, all but a small risk would have been detected. This was an ecological study, and findings from such studies generally need to be treated cautiously because of

Additional references (w1-w4) are on bmj.com

This article was posted on bmi.com on 18 April 2005: http://bmj.com/cgi/doi/10.1136/bmj.38449.476759.AE 